\title{
Dinámica no lineal de la regulación autonómica cardiovascular durante la prueba del peso sostenido en pacientes con diabetes mellitus tipo 2
}

\author{
Nonlinear dynamics of cardiovascular autonomic regulation during the sustained weight \\ test in patients with type 2 diabetes mellitus
}

\author{
Michel Torres-Leyva ${ }^{1}$, Frank Hernández-García ${ }^{2}$, Luis A. Lazo-Herrera ${ }^{3 *}$, Laritza Ortiz-Alcolea ${ }^{1}$ y \\ Miguel E. Sánchez-Hechavarría ${ }^{4,5}$
}

${ }^{1}$ Laboratorio de Ciencias Básicas Biomédicas, Universidad de Ciencias Médicas de Santiago de Cuba, Santiago de Cuba, Cuba; ${ }^{2}$ Universidad de Ciencias Médicas de Ciego de Ávila, Ciego de Ávila, Cuba; ${ }^{3}$ Departamento de Ciencias Básicas Biomédicas, Universidad de Ciencias Médicas de Pinar del Río, Pinar del Río, Cuba; ${ }^{4}$ Departamento de Ciencias Básicas, Facultad de Medicina, Universidad Católica de la Santísima Concepción, Concepción, Chile; ${ }^{5}$ Departamento de Psicología, Facultad de Ciencias de la Salud, Universidad Adventista de Chile, Chillán, Chile

\section{Resumen}

Introducción: La dinámica no lineal de la regulación autonómica cardiovascular a partir de la variabilidad de la frecuencia cardiaca no ha sido previamente estudiada en pacientes con diabetes mellitus tipo 2 mediante la prueba del peso sostenido como inductor de hiperreactividad cardiovascular. Objetivo: Determinar las variaciones en la dinámica no lineal de la regulación autonómica cardiovascular durante la prueba del peso sostenido en pacientes con diabetes mellitus tipo 2. Método: Estudio cuasiexperimental, antes-después, con grupo control, realizado en junio-agosto de 2018 en la Facultad No. 1 de Medicina de Santiago de Cuba. Se trabajó con 15 pacientes que padecían diabetes mellitus tipo 2 sin otra enfermedad asociada y 15 sujetos sanos, a quienes se les aplicó la prueba del peso sostenido. Resultados: En los pacientes con diabetes mellitus, al realizar la prueba del peso sostenido se produjo un aumento significativo en la presión arterial sistólica, diastólica y media, y en la frecuencia cardiaca. En la entropía muestral, parámetro que evalúa la adaptabilidad de los sistemas, se produjo una disminución significativa (2.28 \pm 0.33 vs. $1.83 \pm 0.47 \mathrm{~ms} ; p=0.003143$ ). El valor del estadístico $C$ de la entropía muestral en estado basal quedó establecido en 0.973 , siendo la variable con mayor capacidad predictiva. Conclusiones: Durante la prueba del peso sostenido en los pacientes con diabetes mellitus tipo 2 se produjo una disminución de la complejidad de la regulación autonómica cardiovascular; la entropía muestral basal constituyó el indicador de mayor eficacia en la identificación de alteraciones autonómicas cardiovasculares.

Palabras clave: Frecuencia cardiaca. Sistema nervioso autónomo. Diabetes mellitus. Diabetes mellitus tipo 2.

\section{Abstract}

Introduction: The analysis of non-linear dynamics of cardiovascular autonomic regulation based on heart rate variability has not been previously studied in patients with type 2 diabetes mellitus using the sustained weight test as an inducer of cardiovascular hyperreactivity. Objective: To determine the variations in the non-linear dynamics of cardiovascular autonomic

\section{Correspondencia:}

*Luis A. Lazo-Herrera

E-mail: lazoherrera96@gmail.com
Disponible en internet: 29-11-2021 Rev Colomb Cardiol. 2021;28(5):421-430 www.rccardiologia.com 0120-5633 / @ 2020 Sociedad Colombiana de Cardiología y Cirugía Cardiovascular. Publicado por Permanyer. Este es un artículo open access bajo la licencia CC BY-NC-ND (http://creativecommons.org/licenses/by-nc-nd/4.0/). 
regulation during the sustained weight test in patients with type 2 diabetes mellitus. Method: A quasi-experimental, before-after study with a control group, from June-August 2018, at the No. 1 School of Medicine in Santiago de Cuba. We worked with 15 patients suffering from type 2 diabetes mellitus without other associated disease and 15 healthy subjects, to whom the sustained weight test was applied. Results: In patients with diabetes mellitus, when performing the sustained weight test, there was a significant increase in the hemodynamic parameters TAS, TAD, TAM and FC. In the sample entropy, a parameter that evaluates the adaptability of the systems, there is a significant decrease $(2.28 \pm 0.33 \mathrm{vs}$. $1.83 \pm 0.47 \mathrm{~ms} ; p=0.003143)$. The value of the $C$ statistic of the sample entropy at baseline was established at 0.973 , being the variable with the highest predictive capacity. Conclusions: In patients with type 2 diabetes mellitus during the sustained weight test, there was a decrease in the complexity of cardiovascular autonomic regulation, the basal sample entropy was the indicator of greater efficacy in the identification of cardiovascular autonomic alterations.

Key words: Heart rate. Autonomic nervous system. Diabetes mellitus. Diabetes mellitus type 2.

\section{Introducción}

La variabilidad de la frecuencia cardiaca se considerada un mecanismo de modulación del sistema nervioso autónomo ${ }^{1}$ y se define como la variación de la frecuencia del latido cardiaco durante un intervalo de tiempo definido, nunca superior a 24 horas en un análisis de periodos circadianos consecutivos. Su estudio puede ser útil en el diagnóstico y el tratamiento de las complicaciones de la salud cardiovascular, y también como herramienta complementaria en la prescripción de ejercicio físico para las personas sedentarias, deportistas y con enfermedades del corazón u otras enfermedades tanto físicas como psíquicas. En una persona sana en reposo, los latidos se producen con una frecuencia variable, es decir, el tiempo entre dos latidos varía a lo largo de un registro ${ }^{2}$.

Los métodos no lineales de medir la variabilidad de la frecuencia cardiaca posibilitan un análisis desde la teoría del caos de la complejidad de la regulación de la frecuencia cardiaca ${ }^{3}$. El análisis del comportamiento teórico de los sistemas caóticos puede aportar conceptos nuevos, ya que los seres vivos poseen biorritmos que en estados de buena salud fluctúan de forma aparentemente aleatoria. Uno de los sistemas biológicos analizados es el corazón, el cual se ha venido estudiando con las herramientas de la teoría del caos ${ }^{4}$. Esta teoría establece que los sistemas dinámicos no lineales y complejos son inherentemente impredecibles, pero al mismo tiempo garantiza que, a menudo, la mejor manera de definir el comportamiento de estos sistemas es mediante las representaciones gráficas en el espacio-fase. Es así como la aparición de regularidad en el funcionamiento cardiaco es un signo de alteración, mientras que el mantenimiento de un comportamiento caótico es señal de buen funcionamiento ${ }^{5,6}$.
Las series temporales obtenidas de sistemas naturales como el corazón tienen características comunes, tales como que son irregulares y presentan un espectro de Fourier con una rica variedad de amplitudes; poseen estabilidad global por moverse dentro de un rango determinado y al graficarse estas series en un plano físico despliegan figuras geométricas complejas, denominadas atractores extraños o caóticos ${ }^{5-7}$.

El caos intrínsecamente aprovecha la riqueza relacionada con su estructura, y es por esto que hay beneficios para estos sistemas de la adopción de regímenes caóticos con una amplia gama de conductas posibles ${ }^{7}$. La entropía muestral es uno de los indicadores no lineales del balance simpático-vagal en la regulación autonómica del corazón ${ }^{8}$ y cuantifica la regularidad de un sistema, de forma que cuanto más predecible es una serie, menor es el valor de la entropía, por lo que se reduce con el envejecimiento, al igual que los sistemas patológicos muestran entropías menores que los sistemas sanos ${ }^{6}$, que desde la perspectiva de la teoría de la complejidad se pueden interpretar como una pérdida de la adaptabilidad de los sistemas reguladores del ritmo cardiaco.

La diabetes mellitus es una enfermedad endocrinometabólica crónica, caracterizada por una elevación de la glucosa en sangre (hiperglucemia) como consecuencia de una deficiencia absoluta en la producción por parte del organismo de la hormona insulina, o una incapacidad para utilizar efectivamente la que produ$\mathrm{ce}^{9,10}$. Hay tres tipos principales de diabetes: tipo 1, tipo 2 (DMT2) y diabetes gestacional; de ellas, la DMT2 es la más común. Dadas las altas incidencia y prevalencia de la diabetes mellitus, así como la carga económica y sanitaria que genera, esta enfermedad se ha convertido en un problema para la salud pública mundial ${ }^{10-13}$.

En los pacientes con DMT2 puede aparecer neuropatía autonómica cardiaca, que produce alteraciones 
de la presión arterial y de la variabilidad de la frecuencia cardiaca. El test de fuerza de agarre (handgrip) es una herramienta común para su estudio, pero representa una dificultad en pacientes de edad avanzada, sobre todo en aquellos con artritis en la mano y que tengan pérdida de la fuerza muscular. Por otra parte, está la prueba del peso sostenido (PPS), la cual es una variante del test de handgrip que se utiliza también para inducir hiperreactividad cardiovascular. Esta prueba se desarrolló en la Universidad de Ciencias Médicas de Villa Clara en los años 1980 y se ha venido aplicando en múltiples investigaciones en el país ${ }^{14}$, pero solo desde la óptica de la variabilidad en sujetos sanos ${ }^{15} y$ con diferentes grados de hiperreactividad ${ }^{16}$, y no se han llevado a cabo estudios en Cuba sobre la variabilidad de la frecuencia cardiaca en pacientes diabéticos mediante esta prueba.

Debido a lo expuesto se decidió realizar este estudio con el objetivo de determinar las variaciones en la dinámica no lineal de la regulación autonómica cardiovascular durante la PPS en pacientes con DMT2.

\section{Método}

\section{Diseño y población del estudio}

Se realizó un estudio no observacional, cuasiexperimental, de tipo antes-después, con grupo control, en el Laboratorio de Ciencias Básicas Biomédicas de la Facultad No. 1 de Medicina de la Universidad de Ciencias Médicas de Santiago de Cuba, durante el periodo de junio-agosto de 2018. Se trabajó con un grupo de estudio formado por 15 pacientes con diagnóstico de DMT2 (6 hombres y 9 mujeres) y uno control con 15 sujetos sanos (8 hombres y 7 mujeres). Todos formaban parte de la población de trabajadores de la institución y no padecían otra enfermedad. Los pacientes con DMT2 cumplían, en su totalidad, tratamiento regular con metformina o hipoglucemiantes orales del tipo sulfonilureas (glibenclamida), con una media de evolución de la enfermedad de $15.4 \pm$ 6.3 años.

\section{Variables}

Las variables objeto de estudio fueron la edad, el peso, la talla, el índice de masa corporal (IMC), la presión arterial sistólica (PAS), la presión arterial diastólica $(P A D)$, la presión arterial media (TAM), la frecuencia cardiaca (FC), el estrés psíquico (SD1), el estrés físico (SD2), la relación SD2/SD1, la entropía muestral, $\alpha, \alpha_{1}$, $\alpha_{2}$ y estados funcionales (reposo y prueba del peso sostenido).

\section{Técnicas y procedimientos}

Se realizó una búsqueda bibliográfica sobre el tema, con vistas a construir el marco teórico de la investigación. Los datos demográficos y clínicos se obtuvieron del interrogatorio y de la exploración física correspondiente.

\section{Procedimientos de medición y registro}

Cada tipo de medición fue registrada por la misma persona, para minimizar errores, en el local de mediciones corporales del Laboratorio de Ciencias Básicas Biomédicas de la Universidad de Ciencias Médicas de Santiago de Cuba. Los participantes estaban en condiciones de ayuno previo (2 horas como mínimo), con la vejiga vacía y sin realizar ejercicio físico alguno ni consumir bebidas alcohólicas en las 12 horas previas a la realización de la prueba. Se pidió a los pacientes cesar su tratamiento 12 horas antes del registro. Se realizaron interrogatorio, exploración física y electrocardiograma de control para descartar cualquier alteración del ritmo cardiaco.

\section{Medidas antropométricas}

La medición de la talla y del peso se realizó con una báscula-tallímetro SOEHNLE Professional ${ }^{\circledR}$ con una precisión de $0,1 \mathrm{~cm}$. La talla se definió como la distancia entre el punto más alto de la cabeza (vértex) y los talones, colocando a los voluntarios de pie, erguidos en posición anatómica y con la cabeza en el plano de Frankfort. Se calculó el IMC.

\section{Registros fisiológicos}

Al comienzo de la sesión de los registros de electrocardiograma, en la mañana (8:30-12:00 a.m.), los sujetos fueron sentados en un sillón confortable, situado en una habitación con temperatura controlada entre 24 y $27^{\circ} \mathrm{C}$, y con luz tenue. En estas condiciones se les permitió reposar 10-15 minutos hasta lograr una mejor adaptación a las condiciones del local, y se procedió a realizar los registros hemodinámicos con la presión arterial y los registros electrofisiológicos autonómicos a través del electrocardiograma; los electrodos se colocaron después de limpiar la piel con alcohol y se ubicaron según las indicaciones del equipo, realizándose las mediciones durante 5 minutos. 
Luego, en posición sentado, se procedió a realizar un ejercicio isométrico que consistió en mantener un peso de 500 gramos con el miembro superior izquierdo extendido en ángulo recto respecto al cuerpo durante 2 minutos. Al cumplirse 1 minuto y 50 segundos del ejercicio se procedió a la toma de la presión arterial en el miembro superior derecho. Se descendió el miembro superior izquierdo a los 2 minutos, y se continuó la medición electrocardiográfica hasta completar 5 minutos. Se realizó un análisis de la variabilidad de la frecuencia cardiaca durante el reposo y en la PPS.

\section{Procesamiento de la señal electrocardiográfica: discriminación de las ondas $R$ y cálculo de los intervalos $R R$}

El procesamiento ulterior de los registros digitalizados incluyó su inspección visual, con el fin de evaluar su calidad y detectar posibles artefactos, así como su edición con la consecuente eliminación de los artefactos encontrados.

La discriminación de los picos $R$ de la señal digitalizada y el cálculo de los intervalos RR se realizaron empleando el método de Sabarimalai-Manikandan y Soman ${ }^{17}$. El conjunto de intervalos RR obtenido fue almacenado y constituye la serie de datos a partir de la cual se realizó todo el análisis posterior de la variabilidad de la frecuencia cardiaca.

\section{Preprocesamiento de los intervalos RR y análisis de la variabilidad de la frecuencia cardiaca}

La cuantificación de la variabilidad de la frecuencia cardiaca en reposo y durante la PPS se efectuó con el programa HRVAS ${ }^{\circledR}$ (https://sourceforge.net/projects/hrvas/) de Ramshur ${ }^{18}$, que permitió realizar el preprocesamiento de las series de intervalos RR y el cálculo de los indicadores no lineales. En el preprocesamiento de la serie de intervalos RR se utilizó un filtro de porcentaje con valor del $20 \%$ del intervalo previo para detectar los latidos ectópicos. El reemplazo de los intervalos ectópicos se hizo a partir de la interpolación cúbica politómica.

\section{Procesamiento de los datos}

Los datos fueron procesados con el paquete estadístico SPSS 21.0 y los resultados se expresan como valores medios y desviación estándar para las variables a las cuales se realizó un análisis estadístico no paramétrico con la prueba $U$ de Mann-Whitney para muestras independientes, comparando los pacientes sanos con los diabéticos (comparación entre grupos). Se realizó también un análisis estadístico no paramétrico con la prueba de los rangos con signo de Wilcoxon para muestras relacionadas (comparación intragrupo), con un nivel de significación de $p<0.05$. Se valoró la capacidad discriminatoria de las variables mediante el estadístico C (área bajo la curva ROC [receiver operating characteristic]).

\section{Consideraciones éticas}

Se respetaron los principios éticos de la Declaración de Helsinki. Se obtuvo la autorización correspondiente para la realización de la investigación. A todos los pacientes incluidos en el estudio se les explicó previamente en qué consistía la prueba a que iban a ser sometidos, sus objetivos y procedimientos, su inocuidad y no interferencia ni modificaciones o retardo en los procedimientos terapéuticos indicados para su enfermedad.

\section{Resultados}

En la tabla 1 se muestran las características de la población de estudio. La edad media de los pacientes con DMT2 fue de $56.67 \pm 8.47$ años y la del grupo control fue de $39.47 \pm 11.86$ años. Desde el punto de vista antropométrico, los pacientes con DMT2 se caracterizaron por un IMC de $29.15 \pm 4.27 \mathrm{~kg} / \mathrm{m}^{2}$, mientras que los sujetos sanos tenían un IMC de $25.27 \pm$ $3.67 \mathrm{~kg} / \mathrm{m}^{2}$, clasificando como sobrepeso el estado nutricional de ambos grupos.

Al evaluar los parámetros hemodinámicos de la variabilidad de la frecuencia cardiaca en los pacientes con DMT2 y los sujetos sanos (Tabla 2), se encontraron diferencias significativas en el estado basal de la PAS $(123.53 \pm 11.79$ vs. $110.67 \pm 11.41 \mathrm{mmHg}$; $\mathrm{p}=0.014342)$ y de la PAM $(108.84 \pm 10.48$ vs. 86.53 $\pm 10.68 \mathrm{mmHg} ; p=0.000073$ ), el SD1 (11.74 \pm 6.63 vs. $21.51 \pm 10.26 \mathrm{~ms} ; p=0.015235)$, el SD2 (29.92 \pm 12.5 vs. $56.81 \pm 19.46 \mathrm{~ms} ; p=0.000391)$ y la entropía muestral $(2.28 \pm 0.33$ vs. $1.67 \pm 0.24 ; p=0.000010)$. Al realizar la PPS en ambos grupos, las diferencias más significativas se hallaron en la PAM (114.04 \pm 12.19 vs. $94.56 \pm 9.23 \mathrm{mmHg} ; p=0.000010$ ), siendo esta mayor durante la PPS en los pacientes diabéticos, el SD1 (12.23 \pm 5.92 vs. $19.35 \pm 8.24 \mathrm{~ms} ; p=0.015224)$, 
Tabla 1. Características de los grupos de estudio

\begin{tabular}{|l|c|c|c|c|c|}
\hline \multirow{2}{*}{ Variable } & \multicolumn{2}{|c|}{$\begin{array}{c}\text { Pacientes con } \\
\text { DMT2 }\end{array}$} & \multicolumn{2}{c|}{ Sujetos sanos } & \multirow{2}{*}{$\mathbf{p}^{*}$} \\
\cline { 1 - 6 } & Media & DE & Media & DE & \\
\hline Edad (años) & 56.67 & 8.47 & 39.47 & 11.86 & 0.000 \\
\hline Peso (kg) & 79.41 & 19.65 & 71.09 & 12.71 & 0.290 \\
\hline Talla (m) & 1.64 & 0.10 & 1.68 & 0.11 & 0.383 \\
\hline IMC (kg/m $)$ & 29.15 & 4.27 & 25.27 & 3.67 & 0.004 \\
\hline
\end{tabular}

DE: desviación estándar; DMT2: diabetes mellitus tipo 2; IMC: índice de masa corporal.

${ }^{*}$ Nivel de significación $\mathrm{p}<0.05$. Prueba $U$ de Mann-Whitney.

el SD2 ( $52.15 \pm 20.89$ vs. $80.21 \pm 27.94 \mathrm{~ms} ; p=0.007010)$ y la entropía muestral $(1.83 \pm 0.47$ vs. $1.33 \pm 0.33$; $p=0.001739)$.

En la tabla 3 se muestra la comparación intragrupos de los estados basal y tras la PPS. Para el grupo de estudio se hallaron diferencias entre ambos estados en los parámetros hemodinámicos PAS $(123.53 \pm 11.79$ vs. $128.26 \pm 13.09 \mathrm{mmHg} ; \mathrm{p}=0.022359), \operatorname{PAD}(79.47 \pm$ 10.51 vs. $84.27 \pm 12.51 \mathrm{mmHg} ; p=0.004864)$, PAM $(108.84 \pm 10.48$ vs. $114.04 \pm 12.19 \mathrm{mmHg} ; p=0.008672)$ y FC (79.62 \pm 13.9 vs. $81.93 \pm 14.04$ I.p.m.; $p=0.035564)$. Igualmente, en el grupo control se encontraron diferencias en la PAS (110.67 \pm 11.41 vs. $119.93 \pm 10.91 \mathrm{mmHg}$; $p=0.001303)$, la $\operatorname{PAD}(74.47 \pm 10.68$ vs. $81.87 \pm$ $8.77 \mathrm{mmHg} ; \mathrm{p}=0.001096)$, la PAM (86.53 \pm 10.68 vs. $94.56 \pm 9.23 \mathrm{mmHg} ; p=0.000951)$ y la FC $(71.97 \pm$ 9.02 vs. $76.68 \pm 8.47$ I.p.m.; $p=0.000655$ ).

Por otro lado, para los parámetros no lineales de la variabilidad de la frecuencia cardiaca, en el grupo de pacientes con DMT2 fueron significativas las diferencias entre el estado basal y tras la PPS para el SD2 (29.92 \pm 12.5 vs. $52.15 \pm 20.89 \mathrm{~ms} ; \mathrm{p}=0.003137)$, la relación SD2/SD1 $(2.98 \pm 0.10$ vs. $5.04 \pm 3.28 \mathrm{~ms}$; $p=0.004514)$, la entropía muestral ( $2.28 \pm 0.33$ vs. 1.83 $\pm 0.47 \mathrm{~ms} ; \mathrm{p}=0.003143)$ y los valores de $\alpha(0.93 \pm$ 0.14 vs. $1.07 \pm 0.19 ; p=0.017059)$ y $\alpha_{2}(0.90 \pm 0.16$ vs. $1.06 \pm 0.21 ; p=0.035564)$. En los sujetos sanos, las variaciones se encontraron en SD1 $(21.51 \pm 10.26$ vs. $19.35 \pm 8.24 \mathrm{~ms} ; p=0.030874)$, SD2 (56.81 \pm 19.46 vs. $80.21 \pm 27.94 \mathrm{~ms} ; p=0.001785)$, SD2/SD1 (2.94 \pm 0.86 vs. $4.41 \pm 1.33 \mathrm{~ms} ; p=0.000805)$, entropía muestral $(1.67 \pm 0.24$ vs. $1.33 \pm 0.33 ; p=0.002324), \alpha(0.94$ \pm 0.16 vs. $1.07 \pm 0.14 ; p=0.008985)$ y $\alpha_{1}(1.12 \pm 0.26$ vs. $1.26 \pm 0.19 ; p=0.023096$ ).

En la tabla 4 se aprecian cinco valores correspondientes a las áreas bajo la curva ROC (fig. 1) cercanos

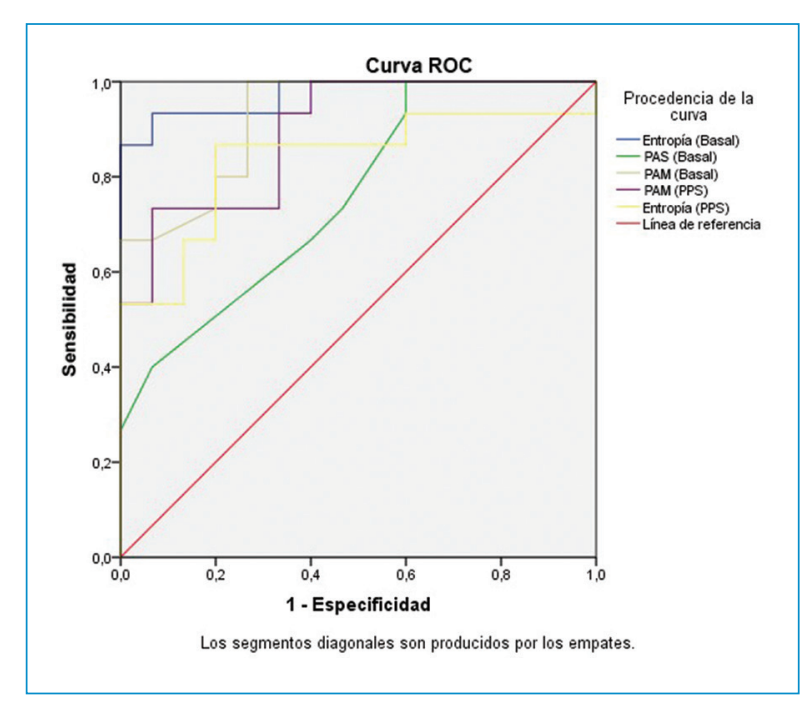

Figura 1. Curva ROC de las variables con capacidad predictiva de alteraciones de la adaptabilidad y la complejidad de la regulación autonómica cardiovascular. PAM: presión arterial media; PAS: presión arterial sistólica; PPS: prueba del peso sostenido.

a 1 (por encima de 0.70), por lo que se puede considerar que esas variables tienen valor predictivo significativo $(p<0.05)$. El valor del estadístico $C$ de la entropía muestral en estado basal quedó establecido en 0.973 , lo cual se traduce en una excelente capacidad predictiva, siendo la de mayor área bajo la curva.

\section{Discusión}

Para el estudio de la variabilidad de la frecuencia cardiaca en pacientes con diagnóstico de DMT2 se han llevado a cabo diversas investigaciones ${ }^{19-22}$, en las cuales ha sido importante la ausencia de grandes diferencias entre las edades y las variables antropométricas de la muestra estudiada, lo cual posibilita la homogenización en la comparación de los pacientes de estudio, reduciendo el sesgo presente por los cambios producidos por estas variables.

Estudios previos ${ }^{23,24}$ han reportado una baja variabilidad de la FC en los pacientes con diabetes mellitus con y sin neuropatía diabética asociada, resultados que coinciden con la investigación realizada. En el estudio existió un incremento significativo de la PAS, la PAD y la PAM, resultados similares a los de Bunsawat y Baynard ${ }^{25}$, además de la FC; también se observó una disminución de la entropía muestral durante el estado basal y la PPS, con resultados similares a los de varios 
Tabla 2. Parámetros hemodinámicos y no lineales de la variabilidad de la frecuencia cardiaca en los estados fisiológicos estudiados entre pacientes con diabetes mellitus tipo 2 y sujetos sanos

\begin{tabular}{|c|c|c|c|c|c|c|}
\hline \multirow[t]{2}{*}{ Variable } & \multirow[t]{2}{*}{ Estado } & \multicolumn{2}{|c|}{ Pacientes con DMT2 } & \multicolumn{2}{|c|}{ Sujetos sanos } & \multirow[t]{2}{*}{$\mathbf{p}^{*}$} \\
\hline & & Media & DE & Media & $\mathrm{DE}$ & \\
\hline \multirow[t]{2}{*}{ PAS (mmHg) } & Basal & 123.53 & 11.79 & 110.67 & 11.41 & 0.014 \\
\hline & PPS & 128.26 & 13.09 & 119.93 & 10.91 & 0.088 \\
\hline \multirow[t]{2}{*}{ PAD $(\mathrm{mmHg})$} & Basal & 79.47 & 10.51 & 74.47 & 10.68 & 0.186 \\
\hline & PPS & 84.27 & 12.51 & 81.87 & 8.77 & 0.393 \\
\hline \multirow[t]{2}{*}{ PAM (mmHg) } & Basal & 108.84 & 10.48 & 86.53 & 10.68 & 0.000 \\
\hline & PPS & 114.04 & 12.19 & 94.56 & 9.23 & 0.000 \\
\hline \multirow[t]{2}{*}{ FC (I.p.m.) } & Basal & 79.62 & 13.9 & 71.97 & 9.02 & 0.064 \\
\hline & PPS & 81.93 & 14.04 & 76.68 & 8.74 & 0.124 \\
\hline \multirow[t]{2}{*}{ SD1 (ms) } & Basal & 11.74 & 6.63 & 21.51 & 10.26 & 0.015 \\
\hline & PPS & 12.23 & 5.92 & 19.35 & 8.24 & 0.015 \\
\hline \multirow[t]{2}{*}{ SD2 (ms) } & Basal & 29.92 & 12.5 & 56.81 & 19.46 & 0.000 \\
\hline & PPS & 52.15 & 20.89 & 80.21 & 27.94 & 0.007 \\
\hline \multirow[t]{2}{*}{ SD2/SD1 } & Basal & 2.98 & 0.10 & 2.94 & 0.86 & 0.708 \\
\hline & PPS & 5.04 & 3.28 & 4.41 & 1.33 & 0.787 \\
\hline \multirow[t]{2}{*}{ Entropía muestral } & Basal & 2.28 & 0.33 & 1.67 & 0.24 & 0.000 \\
\hline & PPS & 1.83 & 0.47 & 1.33 & 0.33 & 0.001 \\
\hline \multirow[t]{2}{*}{$\alpha$} & Basal & 0.93 & 0.14 & 0.94 & 0.16 & 0.917 \\
\hline & PPS & 1.07 & 0.19 & 1.07 & 0.14 & 0.983 \\
\hline \multirow[t]{2}{*}{$\alpha_{1}$} & Basal & 1.12 & 0.27 & 1.12 & 0.26 & 0.950 \\
\hline & PPS & 1.19 & 0.22 & 1.26 & 0.19 & 0.693 \\
\hline \multirow[t]{2}{*}{$\alpha_{2}$} & Basal & 0.90 & 0.16 & 0.91 & 0.19 & 0.755 \\
\hline & PPS & 1.06 & 0.21 & 1.06 & 0.20 & 0.755 \\
\hline
\end{tabular}

DE: desviación estándar; DMT2: diabetes mellitus tipo 2; FC: frecuencia cardiaca; PAD: presión arterial diastólica; PAM: presión arterial media; PAS: presión arterial sistólica; PPS: prueba del peso sostenido; SD1: estrés psíquico; SD2: estrés físico.

*Nivel de significación $\mathrm{p}<0,05$. Prueba U de Mann-Whitney.

autores $^{26-30}$ que reportan que la PPS produce un aumento de la presión arterial y de la FC en sujetos normotensos.

Tanto la prueba de handgrip como la PPS son ejercicios isométricos estáticos, en los que sus respuestas están mediadas por fibras aferentes de los tipos III y IV, activadas por productos del metabolismo anaeróbico y por activación de mecanismos centrales que constituyen el llamado reflejo presor del ejercicio. La respuesta presora al ejercicio produce un incremento de la PAM, la FC y el gasto cardiaco, y un aumento de la resistencia vascular periférica ${ }^{31}$.
Drew $^{32}$ reporta que, como parte de la respuesta presora al ejercicio físico isométrico, se produce una vasoconstricción de la arteria aferente renal, lo cual activa el mecanismo renina-angiotensina-aldosterona que conduce a retención hidrosalina, aumento de la vasoconstricción por la acción de la angiotensina II, mayor resistencia vascular periférica y aumento de la poscarga y de la presión arterial.

La disminución significativa de la entropía muestral en los pacientes con DMT2 al aplicar la PPS, en comparación con el estado de reposo, se debe a que esta constituye un indicador no lineal del 
Tabla 3. Parámetros hemodinámicos y no lineales de la variabilidad de la frecuencia cardiaca entre pacientes con diabetes mellitus tipo 2 y sujetos sanos respecto a los estados fisiológicos estudiados

\begin{tabular}{|c|c|c|c|c|c|c|}
\hline \multirow[t]{2}{*}{ Variables } & \multicolumn{3}{|c|}{ Pacientes con DMT2 } & \multicolumn{3}{|c|}{ Sujetos sanos } \\
\hline & $\begin{array}{c}\text { Basal } \\
(\text { media } \pm \text { DE) }\end{array}$ & $\begin{array}{c}\text { PPS } \\
\text { (media } \pm \mathrm{DE})\end{array}$ & $\mathbf{p}^{*}$ & $\begin{array}{c}\text { Basal } \\
\text { (media } \pm \mathrm{DE})\end{array}$ & $\begin{array}{c}\text { PPS } \\
\text { (media } \pm \mathrm{DE})\end{array}$ & $\mathbf{p}^{*}$ \\
\hline PAS (mmHg) & $123.53 \pm 11.79$ & $128.26 \pm 13.09$ & 0.022 & $110.67 \pm 11.41$ & $119.93 \pm 10.91$ & 0.001 \\
\hline PAD (mmHg) & $79.47 \pm 10.51$ & $84.27 \pm 12.51$ & 0.004 & $74.47 \pm 10.68$ & $81.87 \pm 8.77$ & 0.001 \\
\hline PAM (mmHg) & $108.84 \pm 10.48$ & $114.04 \pm 12.19$ & 0.008 & $86.53 \pm 10.68$ & $94.56 \pm 9.23$ & 0.000 \\
\hline FC (I.p.m.) & $79.62 \pm 13.9$ & $81.93 \pm 14.04$ & 0.035 & $71.97 \pm 9.02$ & $76.68 \pm 8.74$ & 0.000 \\
\hline SD1 (ms) & $11.74 \pm 6.63$ & $12.23 \pm 5.92$ & 0.410 & $21.51 \pm 10.26$ & $19.35 \pm 8.24$ & 0.030 \\
\hline SD2 (ms) & $29.92 \pm 12.5$ & $52.15 \pm 20.89$ & 0.003 & $56.81 \pm 19.46$ & $80.21 \pm 27.94$ & 0.001 \\
\hline SD2/SD1 & $2.98 \pm 0.10$ & $5.04 \pm 3.28$ & 0.004 & $2.94 \pm 0.86$ & $4.41 \pm 1.33$ & 0.000 \\
\hline Entropía muestral & $2.28 \pm 0.33$ & $1.83 \pm 0.47$ & 0.003 & $1.67 \pm 0.24$ & $1.33 \pm 0.33$ & 0.002 \\
\hline$\alpha$ & $0.93 \pm 0.14$ & $1.07 \pm 0.19$ & 0.017 & $0.94 \pm 0.16$ & $1.07 \pm 0.14$ & 0.008 \\
\hline$\alpha_{1}$ & $1.12 \pm 0.27$ & $1.19 \pm 0.22$ & 0.155 & $1.12 \pm 0.26$ & $1.26 \pm 0.19$ & 0.023 \\
\hline$\alpha_{2}$ & $0.90 \pm 0.16$ & $1.06 \pm 0.21$ & 0.035 & $0.91 \pm 0.19$ & $1.06 \pm 0.20$ & 0.069 \\
\hline
\end{tabular}

DE: desviación estándar; DMT2: diabetes mellitus tipo 2; FC: frecuencia cardiaca; PAD: presión arterial diastólica; PAM: presión arterial media; PAS: presión arterial sistólica; PPS: prueba del peso sostenido; SD1: estrés psíquico; SD2: estrés físico.

*Nivel de significación $\mathrm{p}<0.05$. Prueba de los rangos con signo de Wilcoxon.

balance simpático-vagal en la regulación autonómica del corazón, disminuyendo en enfermedades en las que hay un tono simpático aumentado (sensibilidad a la sal ${ }^{33}$, síndrome metabólico, insuficiencia cardiaca) y aumentando en condiciones salutogénicas y de reposo, en las que predomina la actividad parasimpática.

Sin embargo, los cambios en el comportamiento complejo de la regulación dependen del tipo de ejercicio. Weippert, et al. ${ }^{34}$ reportaron en su estudio que el ejercicio dinámico produce un aumento de la entropía, mientras que el estático produce una disminución respecto al reposo; además, encontraron mayores presiones arteriales, resistencias vasculares periféricas y consumo de oxígeno miocárdico durante el ejercicio isométrico estático, respecto al dinámico isotónico.

La disminución de la entropía refleja una pérdida de la complejidad y un aumento del componente simpático durante la prueba, lo que es congruente también con el estudio realizado por Porta, et al. ${ }^{8}$ con la mesa basculante en diferentes grados de inclinación, y por ende de la activación simpática barorrefleja, el cual encontró que, a medida que aumenta la respuesta simpática barorrefleja, disminuye la entropía muestral.
En nuestro estudio se observó un aumento significativo del valor $\alpha$ tras realizar la PPS, lo que indica que hubo un aumento de la suavidad de la geometría fractal $y$, por consiguiente, una pérdida de la fractalidad del fenómeno. El componente $\alpha_{2}$, por otra parte, también presenta un aumento significativo de su valor, lo que plantea un incremento de las oscilaciones lentas del espectro del ritmo cardiaco que se corresponden fisiológicamente con el aumento de la actividad simpático-barorrefleja ante la PPS. El aumento del componente $\alpha_{2}$ influye en el aumento de $\alpha$, lo que se traduce en una disminución de la fractalidad y, por tanto, de la complejidad $^{35}$.

La entropía mide la capacidad de adaptabilidad de los sistemas; a mayor valor de entropía, mayor desorden en los valores de la variabilidad de la frecuencia cardiaca, lo que se asocia a una mejor función cardiovascular. Así, entonces, la capacidad discriminatoria de la entropía basal, al ser mayor que la entropía durante la PPS, se asocia con una mejor función cardiovascular. Esto parte del hecho de que en el estado basal no existe ningún estímulo que obligue al sistema a responder frente a una situación que implique estrés externo, y por ende permite una respuesta más caótica y salutogénica. Como 
Tabla 4. Eficacia de los indicadores hemodinámicos y de la dinámica no lineal de la variabilidad de la frecuencia cardiaca durante el reposo y la prueba del peso sostenido en la identificación de las alteraciones autonómicas cardiovasculares asociadas a la diabetes mellitus tipo 2

\begin{tabular}{|c|c|c|c|c|c|c|c|}
\hline $\begin{array}{l}\text { Variables resultado } \\
\text { de contraste }\end{array}$ & Estado & Punto de corte & Sensibilidad & Especificidad & Índice Youden & Área & $\mathbf{p}^{*}$ \\
\hline \multirow[t]{2}{*}{ FC } & Basal & 72.85 & 0.800 & 0.600 & 0.400 & 0.698 & 0.065 \\
\hline & PPS & 86.55 & 0.467 & 0.933 & 0.400 & 0.664 & 0.125 \\
\hline \multirow[t]{2}{*}{ PAS } & Basal & 105.00 & 1.000 & 0.400 & 0.400 & 0.758 & 0.016 \\
\hline & PPS & 137.50 & 0.400 & 1.000 & 0.400 & 0.680 & 0.093 \\
\hline \multirow[t]{2}{*}{ PAD } & Basal & 79.00 & 0.667 & 0.600 & 0.267 & 0.640 & 0.191 \\
\hline & PPS & 89.00 & 0.533 & 0.733 & 0.266 & 0.589 & 0.407 \\
\hline \multirow[t]{2}{*}{ PAM } & Basal & 94.999 & 1.000 & 0.733 & 0.733 & 0.924 & 0.000 \\
\hline & PPS & 103.6 & 0.733 & 0.933 & 0.666 & 0.893 & 0.000 \\
\hline \multirow[t]{2}{*}{ Entropía muestral } & Basal & 1.997 & 0.867 & 1.000 & 0.867 & 0.973 & 0.000 \\
\hline & PPS & 1.545 & 0.867 & 0.800 & 0.667 & 0.836 & 0.002 \\
\hline \multirow[t]{2}{*}{ SD1 } & Basal & 20.85 & 0.133 & 0.467 & -0.400 & 0.240 & 0.015 \\
\hline & PPS & 13.95 & 0.267 & 0.267 & -0.466 & 0.240 & 0.015 \\
\hline \multirow[t]{2}{*}{ SD2 } & Basal & 44.35 & 0.067 & 0.267 & -0.666 & 0.120 & 0.000 \\
\hline & PPS & 72.90 & 0.133 & 0.400 & -0.467 & 0.211 & 0.007 \\
\hline \multirow[t]{2}{*}{ SD2/SD1 } & Basal & 2.858 & 0.667 & 0.600 & 0.267 & 0.540 & 0.709 \\
\hline & PPS & 4.426 & 0.333 & 0.400 & -0.267 & 0.471 & 0.787 \\
\hline \multirow[t]{2}{*}{$\alpha$} & Basal & 0.858 & 0.800 & 0.400 & 0.200 & 0.489 & 0.917 \\
\hline & PPS & 1.029 & 0.733 & 0.400 & 0.133 & 0.498 & 0.983 \\
\hline \multirow[t]{2}{*}{$\alpha_{1}$} & Basal & 1.301 & 0.333 & 0.800 & 0.133 & 0.507 & 0.950 \\
\hline & PPS & 1.356 & 0.333 & 0.867 & 0.200 & 0.458 & 0.694 \\
\hline \multirow[t]{2}{*}{$\alpha_{2}$} & Basal & 0.73 & 0.867 & 0.333 & 0.200 & 0.467 & 0.756 \\
\hline & PPS & 0.961 & 0.800 & 0.467 & 0.267 & 0.533 & 0.756 \\
\hline
\end{tabular}

DE: desviación estándar; FC: frecuencia cardiaca; PAD: presión arterial diastólica; PAM: presión arterial media; PAS: presión arterial sistólica; PPS: prueba del peso sostenido; SD1: estrés psíquico; SD2: estrés físico.

*Nivel de significación $\mathrm{p}<0.05$

contrapartida de esto, tenemos la PPS (estrés externo) como inductor de hiperreactividad cardiovascular, lo que impondrá una mayor rigidez al sistema, dificultando su adaptación y disminuyendo su aparente aleatoriedad. Las demás variables (PAS basal, PAM basal y PAM durante la PPS) también tienen, en menor medida, capacidad discriminatoria al asociarse a la PPS.

Pueden haber influido en los resultados las diferencias de edad entre el grupo de estudio y el grupo control; no así en relación al IMC, pues pesar de las diferencias entre ambos, las muestras se caracterizan como sobrepeso. El sexo fue una variable de confusión que no se consideró en esta investigación, pues los grupos fueron pareados en cuanto a sexo.

En conclusión, en los pacientes con DMT2 ocurrió una disminución de la complejidad de la regulación autonómica cardiovascular y, por tanto, una capacidad de respuesta adaptativa inferior a la de los sujetos sanos, de modo que la entropía muestral constituye el indicador de mayor eficacia en la identificación de alteraciones autonómicas cardiovasculares asociadas a la DMT2, tanto en el estado de reposo como en la respuesta simpática observada en la PPS. 


\section{Financiamiento}

Los autores declaran no haber recibido ningún financiamiento para la realización de este trabajo.

\section{Conflicto de intereses}

Los autores declaran no tener ningún conflicto de intereses.

\section{Responsabilidades éticas}

Protección de personas y animales. Los autores declaran que los procedimientos seguidos se conformaron a las normas éticas del comité de experimentación humana responsable y de acuerdo con la Asociación Médica Mundial y la Declaración de Helsinki.

Confidencialidad de los datos. Los autores declaran que han seguido los protocolos de su centro de trabajo sobre la publicación de datos de pacientes.

Derecho a la privacidad y consentimiento informado. Los autores han obtenido el consentimiento informado de los pacientes y/o sujetos referidos en el artículo. Este documento obra en poder del autor de correspondencia.

\section{Bibliografía}

1. Rodrigues Jhennyfer AL, Ferrari Gustavo D, Fernandes Igor A, Ferezin Letícia $P$, Trapé Átila A, Bueno Júnior CR. Caracterização da variabilidade da frequência cardíaca em indivíduos com síndrome metabólica. Rev Bras Med Esporte. 2017;23:208-12. [Citado 14 jun 2019]. Disponible en: http://www.scielo.br/scielo.php?script=sci_arttext\&pi$d=S 1517-86922017000300208 \&$ Ing =en.

2. Amat Macías IM, Sarabia Cachadiña E, Naranjo Orellana J. Variabilidad de la frecuencia cardíaca en relación con el ciclo menstrual: revisión. Rev Andal Med Deporte. 2015;8:176. [Citado 14 jun 2019]. Disponible en: http://scielo.isciii.es/scielo.php?script=sci_arttext\&pi$\mathrm{d}=$ S1888-75462015000400012\&lng=es.

3. Sassi R, Cerutti S, Lombardi F, Malik M, Huikuri HV, Peng CK, et al. Advances in heart rate variability signal analysis: joint position statement by the e-Cardiology ESC Working Group and the European Heart Rhythm Association co-endorsed by the Asia Pacific Heart Rhythm Society. EP Europace. 2015;17:1341-53. [Citado 20 may 2019]. Disponible en: https://academic.oup.com/europace/article/17/9/1341/627516.

4. Martínez-Lavín M. Caos, complejidad y cardiología. Arch Cardiol Mex. 2012;82:54-8. [Citado 21 jun 2019]. Disponible en: http://www.scielo.org. $\mathrm{mx} /$ scielo.php?script=sci_arttext\&pid=S1405-99402012000100009\&In$\mathrm{g}=\mathrm{es}$.

5. Wu GQ, Arzeno NM, Shen LL, Tang DK, Zheng DA, Zhao NQ, et al. Chaotic signatures of heart rate variability and its power spectrum in health, aging and heart failure. PLoS One. 2009;4:e4323. [Citado 21 jun 2019]. Disponible en: https://journals.plos.org/plosone/article?id=10.1371/ journal.pone.0004323

6. Naranjo Orellana J, De La Cruz Torres B. La entropía y la irreversibilidad temporal multiescala en el análisis de sistemas complejos en fisiología humana. Rev Andal Med Deporte. 2010;3:29-32. [Citado 21 jun 2019]. Disponible en: https://www.elsevier.es/es-revista-revista-andaluza-medicina-del-deporte-284-pdf-X1888754610478025.

7. García-Cuesta JT, Valencia Arias A, Gutiérrez Betancur V. Caos en la actividad cardíaca desde los sistemas complejos. Ninth LACCEI Latin American and Caribbean Conference (LACCEl'2011). Medellín, Colombia; 2011. [Citado 21 jun 2019]. Disponible en: http://www laccei.org/LACCEI2011-Medellin/ExtendedAbstracts/BI052_Garcia_ EA.pdf.
8. Porta A, Gnecchi-Ruscone T, Tobaldini E, Guzzetti S, Furlan R, Montano N. Progressive decrease of heart period variability entropy based complexity during graded head-up tilt. J Appl Physiol. 2007;103:1143-9. [Citado 14 jun 2019]. Disponible en: https://www.physiology.org/doi/ full/10.1152/japplphysiol.00293.2007.

9. Pérez Rodríguez A, Berenguer Gouarnaluses M. Algunas consideraciones sobre la diabetes mellitus y su control en el nivel primario de salud. MEDISAN. 2015;19:375-90. [Citado 14 jun 2019]. Disponible en: http:// scielo.sld.cu/scielo.php? script=sci_arttext\&pid=S1029-3019201 $5000300011 \& \operatorname{lng}=\mathrm{es}$.

10. Hernández Rodríguez J, Domínguez Yuri A, Mendoza Choqueticlla J. Efectos benéficos del ejercicio físico en las personas con diabetes mellitus tipo 2. Rev Cubana Endocrinol. 2018;29:1-18. [Citado 14 jun 2019]. Disponible en: http://scielo.sld.cu/scielo.php?script=sci_arttext\&pi$\mathrm{d}=$ S1561-29532018000200008\&Ing=es.

11. van der Kooi A-LLF, Snijder MB, Peters RJG, van Valkengoed IGM. The association of handgrip strength and type 2 diabetes mellitus in six ethnic groups: an analysis of the HELIUS study. PLoS One. 2015;10:e0137739. [Citado 14 jun 2019]. Disponible en: https://journals. plos.org/plosone/article?id=10.1371/journal.pone.0137739.

12. Arnold Domínguez Y, González Hernández $\mathrm{O}$, Martínez Vázquez N, Formental Hidalgo BI, de Lourdes Arnold AM, González Calero TM, et al. Incidencia de la diabetes mellitus en Cuba, según tipo, en menores de 18 años de edad. Rev Cubana Endocrinol. 2017;28:1-19. [Citado 14 jun 2019]. Disponible en: http://scielo.sld.cu/scielo.php?script=sci_arttext\&pi$\mathrm{d}=S 1561-29532017000300006 \& \operatorname{lng}=\mathrm{es}$.

13. International Diabetes Federation. IDF Diabetes Atlas, $9^{\text {th }}$ ed. Brussels, Belgium: International Diabetes Federation; 2019.

14. Rodríguez Pena A, Guirado Blanco O, González Paz HJ, Ballesteros Hernández M. Hemodynamics patterns at rest and during isometric sustained weight test in normorreactive, hyperreactive and with hypertensive response young people: gender differences. Rev Cuban Invest Biomed. 2019;38(1). [Citado 18 jun 2019]. Disponible en: http://www.revibiomedica.sld.cu/index.php/ibi/article/view/165/html.

15. Torres-Leyva M, Carrazana-Escalona R, Ormigó-Polo LE Ricardo-Ferro BT, López-Galán E, Ortiz-Alcolea L, et al. Respuesta autonómica cardiovascular durante la prueba isométrica cubana del peso sostenido. CorSalud. 2019;11:1-10. [Citado 22 jun 2019]. Disponible en: http://www.revcorsalud.sld.cu/index.php/cors/article/view/342/812.

16. Rodríguez Pena A, Guirado Blanco O, González Paz HJ, Ballesteros Hernández M, Casas Blanco JC, Cárdenas Rodríguez AE. Balance autonómico basal y durante el ejercicio isométrico en jóvenes con diferente reactividad cardiovascular. CorSalud. 2019;11:11-20. [Citado 22 jun 2019]. Disponible en: http://www.revcorsalud.sld.cu/index.php/cors/article/view/435/813.

17. Sabarimalai-Manikandan M, Soman KP. A novel method for detecting R-peaks in electrocardiogram (ECG) signal. Biomed Signal Process Control. 2012;7:118-28. [Citado 21 jun 2019]. Disponible en: https://doi.org/10.1016/j.bspc.2011.03.004.

18. Ramshur JT. Design, evaluation, and application of heart rate variability analysis software (HRVAS) [Tesis]. Memphis: The University of Memphis; 2010. [Citado 13 jun 2019]. Disponible en: https://doi.org/10.13140/ RG.2.2.33667.81444.

19. Istenes I, Körei AE, Putz Z, Németh N, Martos T, Keresztes K, et al. Heart rate variability is severely impaired among type 2 diabetic patients with hypertension. Diabetes Metab Res Rev. 2014;30:305-12. [Citado 12 ene 2019]. Disponible en: https://onlinelibrary.wiley.com/doi/abs/10.1002/ dmrr.2496.

20. Vukomanovic V, Tadic M, Suzic-Lazic J, Kocijancic V, Celic V. The relationship between heart rate variability and left ventricular layer-specific deformation in uncomplicated diabetic patients. Int J Cardiovasc Imaging. 2017;33:481-90. [Citado 12 ene 2019]. Disponible en: https://link.springer.com/article/10.1007/s10554-016-1023-9.

21. Benichou T, Pereira B, Mermillod M, Tauveron I, Pfabigan D, Maqdasy S, et al. Heart rate variability in type 2 diabetes mellitus: a systematic review and meta-analysis. PLoS One. 2018;13:e0195166. [Citado 12 ene 2019]. Disponible en: https://doi.org/10.1371/journal.pone.0195166.

22. Ortiz-Alcolea L, Cutiño-Clavel I, Rizo-Rodríguez RR, Lazo Herrera LA, Albarrán-Torres FA, Ibarra-Peso JM, et al. Regulación autonómica cardiovascular durante la prueba del peso sostenido en pacientes sanos y diabéticos tipo 2. Rev Cuban Invest Biomed. 2020;39:e361. [Citado 16 abr 2020]. Disponible en: http://www.revibiomedica.sld.cu/index.php/ibi/article/view/361.

23. Cherkas A, Abrahamovych O, Golota S, Nersesyan A, Pichler C, Serhiyenko $\mathrm{V}$, et al. The correlations of glycated hemoglobin and carbohydrate metabolism parameters with heart rate variability in apparently healthy sedentary young male subjects. Redox Biol. 2015;5:301-7. [Citado 12 ene 2019]. Disponible en: https://www.ncbi.nlm.nih.gov/pmc/articles/ PMC4484544/.

24. Cos FX, Barengo NC, Costa B, Mundet-Tudurí X, Lindström J, Tuomilehto JO; DE-PLAN Study Group. Screening for people with abnormal glucose metabolism in the European DE-PLAN Project. Diabetes Res Clin Pract. 2015;109:149-56. [Citado 12 ene 2019]. Disponible en: https:// www.ncbi.nlm.nih.gov/pubmed/25931281. 
Rev Colomb Cardiol. 2021;28(5)

25. Bunsawat K, Baynard T. Cardiac autonomic modulation and blood pressure responses to isometric handgrip and submaximal cycling exercise in individuals with down syndrome. Clin Auton Res. 2016;26:253-60. [Citado 12 ene 2019]. Disponible en: https://www.ncbi.nlm.nih.gov/pubmed/27165540.

26. Ballesteros-Hernández $\mathrm{M}$, Guirado Blanco $\mathrm{O}$, Alfonso-Rodríguez J, Marrero-Martínez JA, Fernández-Caraballo D, Heredia-Ruiz D. Concentraciones de oligoelementos y reactividad vascular en mujeres en edades fértiles y posmenopáusicas. Medicent Electron. 2017;21:316-22. [Citado 12 ene 2019]. Disponible en: http://scielo.sld.cu/scielo.php?script=sci_arttext\&pid=S1029-30432017000400005.

27. León-Regal M, Benet-Rodríguez M, Mass-Sosa L, Willians-Serrano S González-Otero L, León-Valdés A. La hiperreactividad cardiovascular como factor predictivo de la hipertensión arterial en la mujer. Medisur. 2016;14(3). [Citado 12 ene 2019]. Disponible en: http://medisur.sld.cu/ index.php/medisur/article/view/3095/2051.

28. Sánchez-Hechavarria ME, Rodríguez-Gutiérrez LC, Rodríguez-Nuviola J, Ortiz-Alcolea L, Sarmiento-González S. Relación entre la actividad física extraescolar y la hiperreactividad cardiovascular en adolescentes. Rev Cub Med Dep Cul Fis. 2016;11(1).

29. Paz Basanta H, Ventura Espina JL, Rojas Rodríguez I, Rivero de la Torre JR, González Paz H, Menéndez Carrasco J. Valor de la prueba del peso sostenido para pesquisajes de hipertensión arterial a la población. Medicent Electron. 1997;1(2). [Citado 12 ene 2019]. Disponible en: http://www.medicentro.sld.cu/index.php/medicentro/article/ view/9/9
30. Sánchez-Hechavarria M, Carrazana-Escalona R, Ricardo-Ferro B, Cutino-Clavel I, López-Galan E. Autonomic cardiovascular response during the isometric Cuban test of the sustained weight. FASEB J. 2017;31:708 7. [Citado 12 ene 2019]. Disponible en: https://www.fasebj.org/doi/ abs/10.1096/fasebj.31.1_supplement.708.7.

31. Watanabe K, Ichinose M, Tahara R, Nishiyasu T. Individual differences in cardiac and vascular components of the pressor response to isometric handgrip exercise in humans. Am J Physiol Heart Circ Physiol. 2014:306:251-60. [Citado 12 ene 2019]. Disponible en: https://www.ncbi.nlm.nih.gov/pubmed/24213616.

32. Drew RC. Baroreflex and neurovascular responses to skeletal muscle mechanoreflex activation in humans: an exercise in integrative physiology. Am J Physiol Regul Integr Comp Physiol. 2017;313:R654-9. [Citado 12 ene 2019]. Disponible en: https://www.ncbi.nlm.nih.gov/pmc/articles/ PMC5814696/.

33. Fares SA, Habib JR, Engoren MC, Badr KF, Habib RH. Effect of salt intake on beat-to-beat blood pressure nonlinear dynamics and entropy in salt-sensitive versus salt-protected rats. Physiol Rep. 2016;4:e12823. [Citado 12 ene 2019]. Disponible en: https://www.ncbi.nlm.nih.gov/pmc/ articles/PMC4908498/.

34. Weippert M, Behrens M, Gonschorek R, Bruhn S, Behrens K. Muscular contraction mode differently affects autonomic control during heart rate matched exercise. Front Physiol. 2015:6:156. [Citado 12 ene 2019]. Disponible en: https://www.ncbi.nlm.nih.gov/pmc/articles/PMC4436571/.

35. Rajendra Acharya U, Faust O, Adib Kadri N, Suri JS, Yu W. Automated identification of normal and diabetes heart rate signals using nonlinear measures. Comput Biol Med. 2013;43:1523-9. [Citado 12 ene 2019]. Disponible en: https://doi.org/10.1016/j.compbiomed.2013.05.024. 